\title{
Applied Continuous - Time Self - Tuning Control
}

\author{
MAREK KUBALČÍK, VLADIMÍR BOBÁL \\ Department of Process Control \\ Tomas Bata University in Zlín \\ Nad Stráněmi 4511, 76005, Zlín \\ CZECH REPUBLIC \\ kubalcik@fai.utb.cz,bobal@fai.utb.cz \\ http://web.fai.utb.cz/
}

\begin{abstract}
One approach to a recursive identification of continuous - time systems was implemented in self - tuning control of a system of interconnected tanks. Since derivatives of input and output variables of continuous - time systems can not be directly measured, differential filters and filtered variables are established to substitute primary variables. The filtered variables are then used in a recursive identification procedure where the classical recursive least squares method is used to identify the system. Results of real - time experiments are compared to results obtained with an analogical discrete controller.
\end{abstract}

Key-Words: - self - tuning control, adaptive control, recursive estimation, nonlinear systems, polynomial methods

\section{Introduction}

A significant part of technological processes in industrial practice has a stochastic character. Traditional controllers with fixed parameters are often unsuitable for such processes, because parameters of the process vary in time and control can not be optimal. A possible method for control of systems with variable parameters is adaptive control using self - tuning controllers [1]. Another wide range of processes belong to a class of nonlinear systems. A nonlinear dynamic behaviour often causes conventional control methods to be unsatisfactory. This problem can be also solved by using self - tuning control. Self - tuning controllers are based on recursive identification of an unknown process on the basis of input and output variables and consequent synthesis of a controller. This approach had beginning in [2], where a special - purpose computer for an identification of parameters of a linear model with subsequent computation of a control law based on minimization of the quadratic criterion was proposed. The part of the recursive identification is as important as the part of a synthesis of a controller. For purposes of the adaptive control only those methods of identification are interesting, which are realizable in real - time.

In this paper, application of one approach to recursive identification of continuous - time system parameters is presented. Since input and output derivatives of a system can not be directly measured, the differential filters and filtered variables are established to substitute primary variables. This approach is described in detail in [3], [4], [5]. The filtered variables are then used in the recursive identification procedure, where the classical recursive least squares method is used to identify the parameters.
Whilst methods of design and applications of discrete self tuning controllers are frequently presented in literature, for example [6], self - tuning control of continuous - time systems is not widespread and there still is not much experience with continuous - time system parameter estimation. Limitations during control of systems with fast dynamics when discrete models with small sampling periods have poor numerical attributes are mostly solved using delta models [7]. An alternative solution is using of the method applied in this paper when a continuous time model is recursively estimated and design of a controller is performed in the continuous - time domain. This approach enables fast sampling. The value of the sampling period is then dependant only on capabilities of used hardware and software. The used software must enable realization of filters by differential equations.

A continuous time self - tuning controller with 2 dof (two degree of freedom) configuration, when a controller contains both feedback and feedforward parts [8], was implemented for real - time control of a model of interconnected tanks. The model is a nonlinear system with variable parameters. The results of control were compared to results obtained with an analogical self tuning discrete controller. Design of the controllers was based on polynomial methods [9].

\section{Design of Controllers}

A model of the controlled system is supposed to be expressed by a transfer function

$$
G(q)=\frac{b(q)}{a(q)}
$$


where $q=s$ for a continuous - time system and $q=z^{-1}$ for a discrete system

A model of the second order which is widely applied in practice and has proved to be effective for control of a range of various processes was chosen for description of dynamic behaviour of the further described system of interconnected tanks in the neighbourhood of a steady state. The polynomials $a$ and $b$ have the following forms in case of the continuous-time model

$$
a(s)=s^{2}+a_{1} s+a_{0}, b(s)=b_{0}
$$

and

$$
a\left(z^{-1}\right)=1+a_{1} z^{-1}+a_{2} z^{-2}, b\left(z^{-1}\right)=b_{1} z^{-1}+b_{2} z^{-2}
$$

in case of the discrete model. In both cases the polynomials $a$ and $b$ are supposed to be coprime.

Differential equation of the continuous - time system is given by

$$
y^{\prime \prime}+a_{1} y^{\prime}+a_{0} y=b_{0} u
$$

and difference equation of the discrete system by

$$
y(k)=-a_{1} y(k-1)-a_{2} y(k-2)+b_{1} u(k-1)+b_{2} u(k-2)
$$

The 2dof configuration of the closed loop with both feedback and feedforward parts was chosen. It is depicted in Fig. 1.

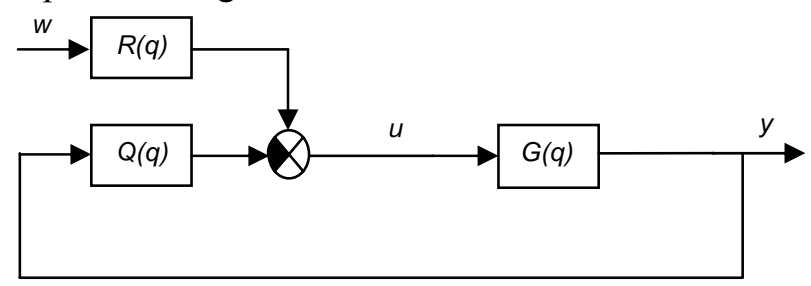

Fig. 1. The 2dof control system configuration.

The basic general requirements on the control system are:

- Internal properness and stability of the control system

- Asymptotic tracking of a reference

The controller has two parts: a feedback part and a feedforward part. The feedback part is defined as

$$
Q(q)=\frac{q(q)}{p(q)}
$$

and the feed - forward part as

$$
R(q)=\frac{r(q)}{p(q)}
$$

Where $q, p$ and $r, p$ are coprime polynomials.

The reference signal can be also described as a ratio of two polynomials

$$
W(q)=\frac{h_{w}(q)}{f_{w}(q)}, \operatorname{deg} h_{w} \leq \operatorname{deg} f_{w}
$$

For particular signals in the control loop can be derived following expressions in the complex domain (operator $q$ will be omitted from some expressions for the purpose of simplification):

Controlled variable:

$$
Y=\frac{1}{a p+b q}\left[b r \frac{h_{w}}{f_{w}}\right]
$$

Control error:

$$
E=\frac{1}{a p+b q}\left[(d-b r) \frac{h_{w}}{f_{w}}\right]
$$

Manipulated variable:

$$
U=\frac{r}{p} \frac{h_{w}}{f_{w}}\left(1-\frac{q b}{a p+b q}\right)
$$

Stability of the closed loop is ensured by the feedback controller $Q$ by solving equation

$$
d=a p+b q
$$

where $d$ is a stable desired polynomial. Its poles are the ruling factors for the behaviour of the closed loop system.

Internal properness is ensured when the following conditions are fulfilled: $\operatorname{deg} q \leq \operatorname{deg} p \quad \operatorname{deg} r \leq \operatorname{deg} p$ To fulfill the requirement on the asymptotic tracking the denominator of the reference signal must be eliminated from the expression for the permanent control error.

$$
\lim _{s \rightarrow 0}[s \cdot E(s)]=\lim _{s \rightarrow 0}\left[s \frac{1}{d}\left[(d-b r) \frac{h_{w}}{f_{w}}\right]\right]=0
$$

From the expression (13) it is obvious that the adequate condition of the asymptotic tracking is divisibility of the polynomial $d-b r$ by the polynomial $f_{w}$. The following Diophantine equation must be then fulfilled

$$
d-b r=t f_{w}
$$

where $t$ is an unknown additional polynomial.

The resulting controller is given by solution of two Diophantine equations:

$$
a p+b q=d \quad t f_{w}+b r=d
$$

Further it is necessary to determine degrees of particular polynomials. Relations for their computation are given by the requirement so that number of unknown controller's parameters and number of algebraic equations resulting from the Diophantine equations are equal. The requirement on the properness of the controller must be also fulfilled.

$$
\operatorname{deg} q=\operatorname{deg} a-1 \quad \operatorname{deg} p \geq \operatorname{deg} a-1+k
$$

where

$$
\begin{aligned}
& k=\operatorname{deg} f_{w}-\operatorname{deg} a \\
& k=0 \text { for } \operatorname{deg} f_{w}-\operatorname{deg} a \leq 0 \\
& \operatorname{deg} r=\operatorname{deg} f_{w}-1 \quad \operatorname{deg} t=2 \operatorname{deg} a-1-\operatorname{deg} f_{w}+k \\
& \operatorname{deg} d \geq 2 \operatorname{deg} a-1+k
\end{aligned}
$$

\subsection{Design of Continuous - Time Controller}

The reference was considered to be from a class of step and ramp signals. 
The ramp reference signal $w(t)=w_{0} t$ has the Laplace transform in the form $W(s)=\frac{w_{0}}{s^{2}}=\frac{h_{w}}{f_{w}} . \operatorname{deg} f_{w}=2$ and $k=\operatorname{deg} f_{w}-\operatorname{deg} a=2-2=0 \Rightarrow k=0$. Degrees of the particular polynomials are derived according to expressions (16) and (17).

$\operatorname{deg} q=\operatorname{deg} a-1=2-1=1 \quad k=\operatorname{deg} f_{w}-\operatorname{deg} a=2-2=0$

$\operatorname{deg} p \geq \operatorname{deg} a-1+k=2-1+0=1$

$\operatorname{deg} r=\operatorname{deg} f_{w}-1=2-1=1$

$\operatorname{deg} t=2 \operatorname{deg} a-1-\operatorname{deg} f_{w}+k=4-1-2+0=1$

$\operatorname{deg} d \geq 2 \operatorname{deg} a-1+k=4-1+0=3$

The polynomials then take following forms:

$$
\begin{gathered}
q(s)=q_{0}+q_{1} s \quad p(s)=p_{0}+p_{1} s \quad r(s)=r_{0}+r_{1} s \\
t(s)=t_{0}+t_{1} s \\
d(s)=s^{3}+d_{2} s^{2}+d_{1} s+d_{0}
\end{gathered}
$$

The Diophantine equations (15) define sets of algebraic equations with unknown controller's parameters

$$
\left[\begin{array}{cccc}
0 & 1 & 0 & 0 \\
1 & a_{1} & 0 & 0 \\
a_{1} & a_{0} & 0 & b_{0} \\
a_{0} & 0 & b_{0} & 0
\end{array}\right]\left[\begin{array}{l}
p_{0} \\
p_{1} \\
q_{0} \\
q_{1}
\end{array}\right]=\left[\begin{array}{c}
1 \\
d_{2} \\
d_{1} \\
d_{0}
\end{array}\right]\left[\begin{array}{cccc}
0 & 0 & 0 & 1 \\
0 & 0 & 1 & 0 \\
0 & b_{0} & 0 & 0 \\
b_{0} & 0 & 0 & 0
\end{array}\right]\left[\begin{array}{c}
r_{0} \\
r_{1} \\
t_{0} \\
t_{1}
\end{array}\right]=\left[\begin{array}{c}
1 \\
d_{2} \\
d_{1} \\
d_{0}
\end{array}\right]
$$

The parameters are obtained by solving these equations.

The resulting controllers then take the form

$$
Q(s)=\frac{q(s)}{p(s)}=\frac{q_{1} s+q_{0}}{p_{1} s+p_{0}} \quad R(s)=\frac{r(s)}{p(s)}=\frac{r_{1} s+r_{0}}{p_{1} s+p_{0}}
$$

The control law can be expressed by following equation

$$
u^{\prime} p_{1}+u p_{0}=r_{1} w^{\prime}+r_{0} w-q_{1} y^{\prime}-q_{0} y
$$

\subsection{Design of Discrete Controller}

The reference signal has $Z$ - transform in the form $W(z)=\frac{w_{0} T_{v} z}{(z-1)^{2}}=\frac{h_{w}}{f_{w}} \quad$ where $\quad T_{v}$ is sampling period.

Degrees of the polynomials are computed according to expressions given in the previous section and the polynomials can be expressed as

$$
\begin{gathered}
p\left(z^{-1}\right)=1+p_{1} z^{-1} \quad q\left(z^{-1}\right)=q_{0}+q_{1} z^{-1} \quad r\left(z^{-1}\right)=r_{0}+r_{1} z^{-1} \\
t\left(z^{-1}\right)=1+t_{1} z^{-1} \quad d\left(z^{-1}\right)=1+d_{1} z^{-1}+d_{2} z^{-2}+d_{3} z^{-3}
\end{gathered}
$$

The sets of algebraic equations with unknown controller's parameters are as follows

$\left[\begin{array}{ccc}1 & b_{1} & 0 \\ a_{1} & b_{2} & b_{1} \\ a_{2} & 0 & b_{2}\end{array}\right]\left[\begin{array}{c}p_{1} \\ q_{0} \\ q_{1}\end{array}\right]=\left[\begin{array}{c}d_{1}-a_{1} \\ d_{2}-a_{2} \\ d_{3}\end{array}\right]\left[\begin{array}{ccc}1 & b_{1} & 0 \\ -2 & b_{2} & b_{1} \\ 1 & 0 & b_{2}\end{array}\right]\left[\begin{array}{c}t_{1} \\ r_{0} \\ r_{1}\end{array}\right]=\left[\begin{array}{c}d_{1}+2 \\ d_{2}-1 \\ d_{3}\end{array}\right]$

The controllers are defined as

$Q\left(z^{-1}\right)=\frac{q\left(z^{-1}\right)}{p\left(z^{-1}\right)}=\frac{q_{0}+q_{1} z^{-1}}{1+p_{1} z^{-1}} R\left(z^{-1}\right)=\frac{r\left(z^{-1}\right)}{p\left(z^{-1}\right)}=\frac{r_{0}+r_{1} z^{-1}}{1+p_{1} z^{-1}}$

The control law takes the form

$u(k)=r_{0} w(k)+r_{1} w(k-1)+q_{0} y(k)+q_{1} y(k-1)-p_{1} u(k-1)(26)$

\section{System Identification}

\subsection{Identification of Discrete Model}

Let The recursive least squares method was used as the basis of our algorithm. We can consider a stochastic process described by an ARX model in the form

$$
a\left(z^{-1}\right) y(k)=b\left(z^{-1}\right) u(k)+e_{s}(k)
$$

which can be expressed in a vector form

$$
y(k)=\boldsymbol{\Theta}^{T}(k) \varphi(k-1)+e_{s}(k)
$$

where $e_{s}$ is an un-measurable random component.

Vector of parameters is defined as

$$
\boldsymbol{\Theta}^{T}(k)=\left[a_{1}, a_{2}, b_{1}, b_{2},\right]
$$

and the regression vector is

$$
\boldsymbol{\varphi}^{T}(k-1)=[-y(k-1),-y(k-2), u(k-1), u(k-2)]
$$

The aim of the identification is a recursive estimation of unknown model parameters $\boldsymbol{\Theta}$ on the basis of the inputs and the outputs considering the time moment $k,\{y(i)$, $\left.u(i), i=k, k-1, k-2, \ldots, k_{0}\right\}$ (where $k_{0}$ is an initial time of the identification). We are looking for a vector $\hat{\boldsymbol{\Theta}}$ minimizing the criterion

$$
J_{k}(\boldsymbol{\Theta})=\sum_{i=k_{0}}^{k} e_{s}^{2}(i)
$$

where

$$
e_{s}(i)=y(i)-\boldsymbol{\Theta}^{T} \phi(i)=\left[\begin{array}{ll}
1 & -\boldsymbol{\Theta}^{T}
\end{array}\left[\begin{array}{l}
y(i) \\
\phi(i)
\end{array}\right]\right.
$$

Tracking of slow changes of the parameters, which is relevant owing to the control of the system of interconnected tanks (nonlinear system with variable parameters), can be achieved by application of exponential forgetting. This technique ensues from the assumption that new data describes the dynamics of an object better than older data, which are multiplied by smaller weighting coefficients. Then we minimize a modified criterion

$$
J_{k}(\boldsymbol{\Theta})=\sum_{i=k_{0}}^{k} \varphi^{2(k-i)} e_{s}^{2}(i)
$$

where $0\left\langle\varphi^{2} \leq 1\right.$ is the exponential forgetting factor. In case that the identified plant is insufficiently activated - it means that the input and output signals are steady (this situation is typical for closed control systems), the exponential forgetting factor can cause numerical instability of the identification algorithm. A possible solution of this problem is application of the adaptive directional forgetting [10]. This technique changes the forgetting factor according to the level of information in the data. In the examples described bellow, the recursive least squares method supported by directional forgetting was applied.

In this case, the vector of parameters is actualised according to the following recursive expression 


$$
\hat{\boldsymbol{\Theta}}(k)=\hat{\boldsymbol{\Theta}}(k-1)+\frac{\boldsymbol{C}(k-1) \varphi(k-1)}{1+\xi(k-1)} \hat{e}(k-1)
$$

where

$$
\xi(k)=\boldsymbol{\varphi}^{T}(k-1) \boldsymbol{C}(k-1) \boldsymbol{\varphi}(k-1)
$$

is an auxiliary scalar and

$$
\hat{e}(k)=y(k)-\hat{\boldsymbol{\Theta}}^{T}(k) \varphi(k-1)
$$

is a prediction error. If $\xi\left(t_{k}\right)>0$, then the square covariance matrix $\boldsymbol{C}$ is actualised according to following expression

$$
\boldsymbol{C}(k)=\boldsymbol{C}(k-1)-\frac{\boldsymbol{C}(k-1) \varphi(k-1) \boldsymbol{\varphi}^{T}(k-1) \boldsymbol{C}(k-1)}{\varepsilon^{-1}(k)+\xi(k-1)}
$$

where

$$
\varepsilon(k)=\varphi(k)-\frac{1-\varphi(k)}{\xi(k-1)}
$$

If $\xi\left(t_{k}\right)=0$, then

$$
\boldsymbol{C}(k)=\boldsymbol{C}(k-1)
$$

The directional forgetting factor is computed in each sampling period according to the expression

$\varphi(k)=\left\{1+(1+\rho)[\ln (1+\xi(k-1))]+\left[\frac{(v(k-1)+1) \eta(k-1)}{1+\xi(k-1)+\eta(k-1)}-1\right] \frac{\xi(k-1)}{1+\xi(k-1)}\right\}$

where

$$
\eta(k)=\frac{\hat{e}^{2}(k)}{\lambda(k)} ; \nu(k)=\varphi(k)\left[(v(k-1)+1] ; \lambda(k)=\varphi(k)\left[\lambda(k-1)+\frac{\hat{e}^{2}(k-1)}{1+\xi(k-1)}\right]\right.
$$

are auxiliary variables.

\subsection{Identification of Continuous -Time Model}

It is not possible to measure directly input and output derivatives of a system in case of continuous - time control loop. One of the possible approaches to this problem is establishing of filters and filtered variables to substitute the primary variables. This approach is described in detail in [3], [4], [5]. The filtered variables are then used in the recursive identification procedure.

Let us consider a linear continuous - time ARX model in a form of differential equation

$$
a(\sigma) y(t)=b(\sigma) u(t)+n(t)
$$

where $n(t)$ is a random continuous - time variable and $\sigma$ is the derivative operator. After the Laplace transform we obtain

$$
a(s) Y(s)=b(s) U(s)+n(s)+o_{1}(s)
$$

where the polynomial $o_{1}$ represents the Laplace transform of initial conditions. The output of the system is than given as

$$
Y(s)=\frac{b(s)}{a(s)} U(s)+\frac{n(s)}{a(s)}+\frac{o_{1}(s)}{a(s)}
$$

In order to obtain approximations of derivatives of the continuous - time variables it is necessary to establish filters using differential equations

$$
c(\sigma) u_{f}(t)=u(t) \quad c(\sigma) y_{f}(t)=y(t)
$$

where $c(\sigma)$ is a stable polynomial and $u_{f}$ is a filtered input and $y_{f}$ is a filtered output. After the Laplace transform we obtain

$$
c(s) U_{f}(s)=U(s)+o_{2}(s) ; \quad c(s) Y_{f}(s)=Y(s)+o_{3}(s)
$$

where $o_{2}(s)$ is a polynomial of initial conditions for the filtered input and $o_{3}(s)$ is a polynomial of initial conditions for the filtered output. The degree of the polynomial $c$ must be greater or equal to the degree of the polynomial $a(\operatorname{deg} c(s) \geq \operatorname{deg} a(s))$. It is profitable to choose $\operatorname{deg} c(s)=\operatorname{deg} a(s)$ (the lower is the degree of the polynomial $c$, the faster is the dynamics of the filter). Time constants of the filters must be lower than time constants of the plant. A right choice of the filter's constants makes convergence of the parameters faster.

After substitution of the filtered variables to the equation (43) we obtain

$$
a\left\lfloor c Y_{f}(s)-o_{3}\right\rfloor=b\left\lfloor c U_{f}-o_{2}\right\rfloor+N(s)+o_{1}
$$

After modification and substitution

$a Y_{f}(s)=b U_{f}(s)+\frac{o_{1}-b o_{2}+a o_{3}+N(s)}{c} o=\frac{o_{1}-b o_{2}+a o_{3}}{c}$

we obtain

$$
Y_{f}(s)=\frac{b}{a} U_{f}(s)+\frac{o}{a}+\frac{1}{a} N(s) \Rightarrow G_{f}(s)=\frac{b}{a}=G(s)
$$

Expression (50) proves that the transfer behaviour between the filtered and between the non - filtered variables is equivalent. Different are only initial conditions for the filtered and original variables. This fact enables to employ the filtered variables for the model parameter estimation.

After transformation to the time domain we obtain the following equation

$$
a(\sigma) y_{f}(t)=b(\sigma) u_{f}(t)+n(t)
$$

The filtered variables are taken in discrete time intervals $t_{k}=k T_{s}, k=0,1,2, \ldots$, where $T_{s}$ is the sampling period. The equation (51) can be modified to the form suitable for the model parameters estimation

$$
y^{(n)}{ }_{f}\left(t_{k}\right)=-\sum_{i=0}^{n-1} a_{i} y_{f}^{(i)}\left(t_{k}\right)+\sum_{j=0}^{m} b_{j} u_{f}^{(j)}\left(t_{k}\right)+n\left(t_{k}\right)
$$

The parameters of the model are estimated by the recursive method described in the previous section according to expressions (34) - (41). For the considered continuous - time model given by expressions (2) and (4) the equation (51) takes following form

$$
y_{f}^{\prime \prime}\left(t_{k}\right)=-a_{1} y_{f}^{\prime}\left(t_{k}\right)-a_{0} y_{f}\left(t_{k}\right)+b_{0} u_{f}\left(t_{k}\right)+n\left(t_{k}\right)
$$

The regression vector and the vector of parameters are

$$
\begin{aligned}
\boldsymbol{\varphi}^{T}\left(t_{k}\right)=\left[-y_{f}^{\prime}\left(t_{k}\right),-y_{f}\left(t_{k}\right), u_{f}\left(t_{k}\right)\right] \\
\boldsymbol{\Theta}^{T}(k)=\left[a_{1}, a_{0}, b_{0}\right]
\end{aligned}
$$

Considering the order of the system, the filters for both variables were chosen to have second order. 


$$
\begin{aligned}
& y_{f}^{\prime \prime}(t)+c_{1} y_{f}{ }^{\prime}(t)+c_{0} y_{f}(t)=y(t) \\
& u_{f}^{\prime \prime}(t)+c_{1} u_{f}^{\prime}(t)+c_{0} u_{f}(t)=u(t)
\end{aligned}
$$

A right choice of the coefficients of the filter's polynomials and choice of the sampling period are the ruling factors for the speed of the parameter's convergence. Time constants of the filters must be lower than time constants of the plant.

\section{Experimental Examples}

\subsection{Model of Interconnected Tanks}

The controllers were verified and compared by real time control of the model of interconnected tanks. Its principal scheme is on the following figure.

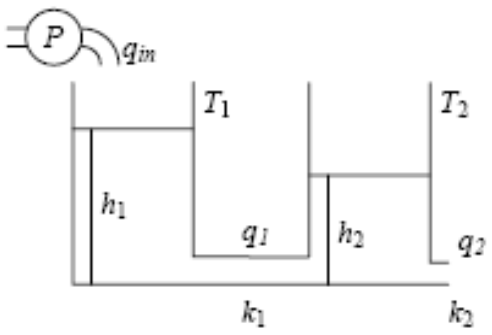

Fig. 2. Scheme of two interconnected tanks.

The system consists of two interconnected cylindrical tanks $T_{1}$ and $T_{2}$ and a pump $P$ which induces inflow to the tank $T_{1}$. The liquid level heights in the tanks $T_{1}$ and $T_{2}$ are $h_{1}$ and $h_{2}$ respectively. The inflow produced by the pump is $q_{\text {in }}$, flow between tanks is $q_{1}$ and the outflow is $q_{2}$. The system can be considered as a single input single output system (SISO) where the input is inflow $q_{i n}$ and output is liquid level $h_{2}$.

The apparatus is a nonlinear system with variable parameters. The nonlinear behaviour is caused by characteristics of the valves, pipes and pumps. Additional nonlinearities are due to air bubbles which are present in the pipes and valves. The bubbles deflate from the pipe system in certain moments. Its description by a linear model is then valid only in a neighbourhood of a steady state. As it was stated in the section 1, selftuning controllers are a possible approach to the control of this kind of system. The nonlinear dynamics are described by a linear model in the neighbourhood of a steady state.

\subsection{Experimental Results}

In case of the continuous-time controller the sampling period for system identification (actualization of the parameters and transposition of the controller) was experimentally assigned as $T_{a}=2 \mathrm{~s}$. The sampling period for actualization of the manipulated variable was assigned $T_{v 1}=0,02 s$. The polynomial $d$ resulted from experiments in the form

$$
d(s)=s^{3}-2,1 s^{2}+1,47 s-0,343
$$

Constants of the filters were chosen to be $c_{0}=0,04 \quad c_{1}=0,4$. Best sampling period for discrete system was found as $T_{v}=2 s$. The polynomial $d$ was chosen as

$$
d\left(z^{-1}\right)=1-1,1805 z^{-1}-0,6390 z^{-2}+0,8195 z^{-3}
$$

Time responses of the control and identified parameters for step and ramp reference signals are in Fig.3 - Fig. 6.

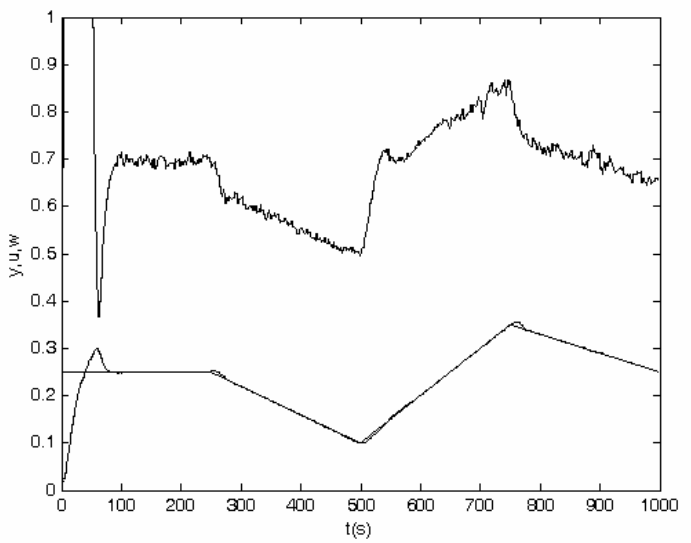

Fig. 3. Control with discrete controller.

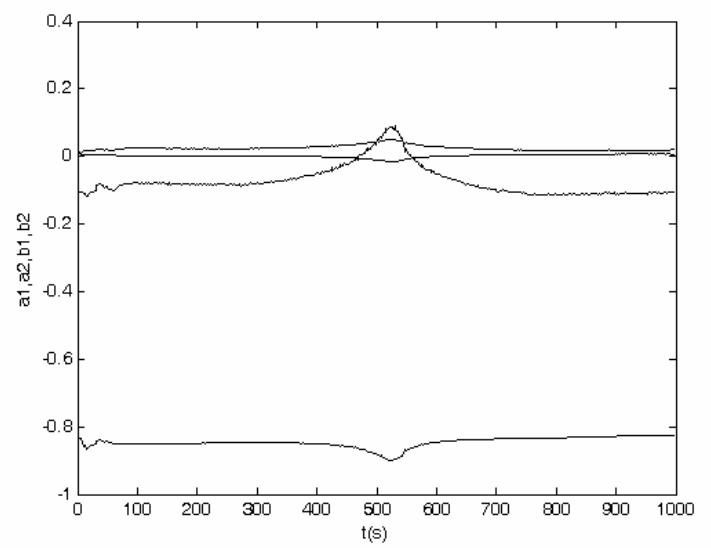

Fig. 4. Control with discrete controller - identified parameters.

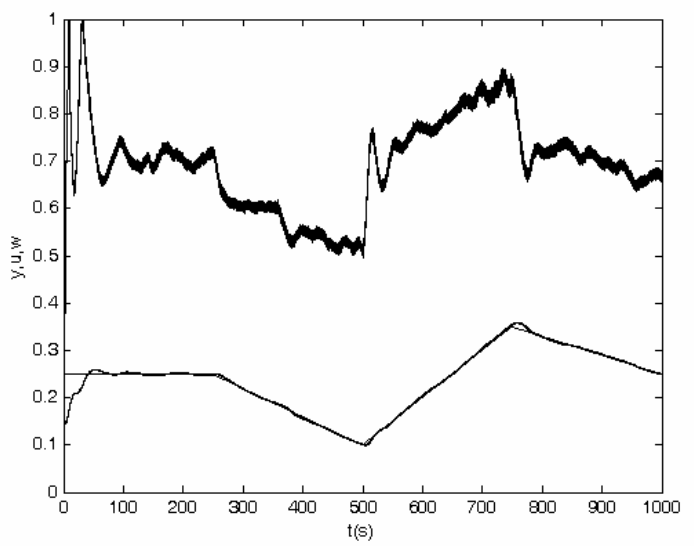

Fig. 5. Control with continuous - time controller. 


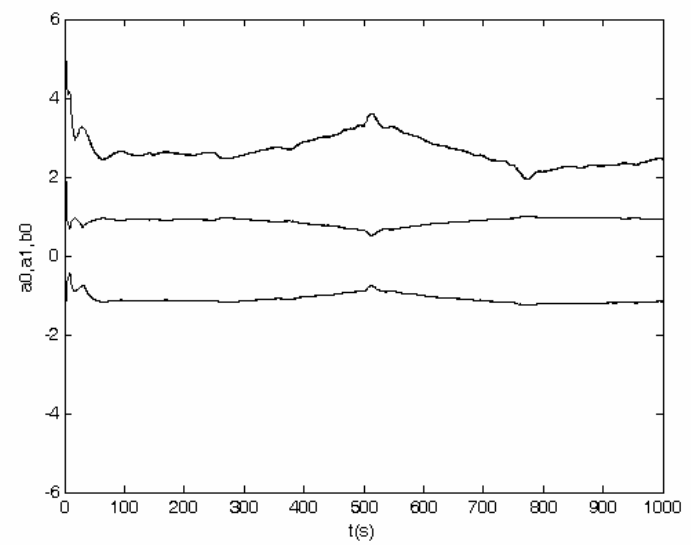

Fig. 6. Control with continuous - time controller identified parameters.

\subsection{Comparison of Control Performance Using Summing Criteria}

The performances of both controllers were compared by means of control quality criteria, which are the sum of powers of tracking errors and the sum of increments of manipulated variables (both sums are related to the number of samples). In case of the continuous - time control the values were taken in discrete time intervals corresponding to sampling of the discrete control $\left(T_{v}=2 \mathrm{~s}\right)$. The Table 1 contains values of the criteria for the entire period of the experiments including the initial part of the parameters estimation. The Table 2 contains values obtained after the parameters fixation

Table 1. Control quality criteria

\begin{tabular}{lcc}
\hline Controller & $\sum e^{2}$ & $\sum \Delta u^{2}$ \\
\hline Continuous - time & 0,1396 & 0,2002 \\
\hline Discrete & 0,7928 & 0,4254 \\
\hline
\end{tabular}

Table 2. Control quality criteria - after parameters fixation

\begin{tabular}{lcc}
\hline Controller & $\sum e^{2}$ & $\sum \Delta u^{2}$ \\
\hline Continuous - time & 0,0032 & 0,0410 \\
\hline Discrete & 0,0078 & 0,0444 \\
\hline
\end{tabular}

\section{Conclusion}

Continuous - time adaptive controller was implemented for control of liquid level of interconnected tanks. The continuous - time model of the controlled system was recursively estimated and design of the controller was performed in the continuous - time domain. This approach enables fast sampling. The value of the sampling period is then dependant only on capabilities of the used hardware and software. The used software must enable realization of filters by differential equations. The applied controller was compared to an analogical discrete controller.

Performances of the discrete and continuous - time controllers were comparable. According to the chosen control quality criteria (Tables 1 and 2) slightly better performed the continuous - time controller. On the other hand, experimental tuning of the continuous - time controller was more complicated. It was rather difficult to find experimentally a suitable conjunction of the tuning parameters. Adjustable parameters are poles of the characteristic polynomial, constants of the filters and the sampling periods $T_{a}$ and $T_{v 1}$. There is a lack of clear theory relating to the closed loop behavior to design parameters. Control courses were rather sensitive to changes of the parameters.

The described method of continuous - time models parameters estimation proved to be effective. A right choice of the filter's constants and the sampling period improves convergence of the parameters. The method is suitable for the identification part of continuous - time self - tuning controllers.

\section{Acknowledgement}

This work was supported in part by the Ministry of Education of the Czech Republic under grant MSM7088352101.

References:

[1] Bobál, V., Bőhm J., Fessl, J., Machacek, J. (2005). Digital self-tuning controllers, Springer - Verlag, London.

[2] Kalman, R. E. (1958). Design of a self optimising control system, Trans. ASME, 80, 1958, pp. 481 492.

[3] Gawthrop, P. J. (1987). Continuous - Time Self Tuning Control, John Willey, Chichester.

[4] Unbehauen, H., Rao, G.P. (1987). Identification of Continuous Systems, North Holland, Amsterdam.

[5] Wahlberg, B. (1990). On the identification of continuous - time dynamical systems, Report LiTHISY-I-0905, Linköping.

[6] Yosof, R., Omatu S., Khaid, M. (1994). Self-tuning PID control: a multivariable derivation and application, Automatica, 30, pp. 1975-1981.

[7] Middleton, R.H., Goodwin, G.C. (1989). Digital Control and Estimation. A Unified Approach. Prentice Hall, Englewood Cliffs, New Jersey.

[8] Grimble, M. J. (1994). Robust Industrial Control. Optimal Design Approach for Polynomial Systems. Prentice Hall, London.

[9] Kučera, V. (1993). Diophantine Equations in Control - a Survey. Automatica, 29, pp. 1361 1375.

[10] Kulhavý, R. (1987). Restricted exponencial forgetting in real - time identification. Automatica, 23 , pp. $589-600$. 